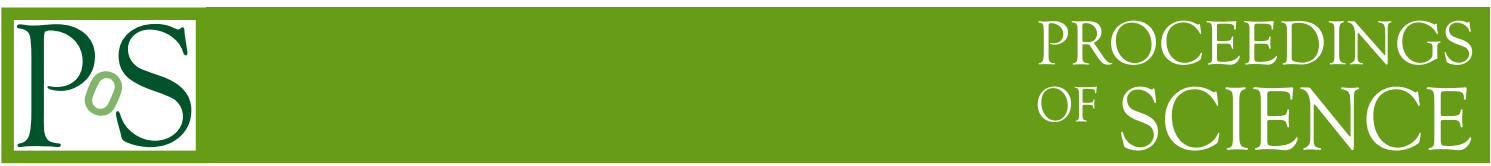

\title{
The cosmic rays web monitor of the LAGO project
}

\section{Otiniano*, Ch. Alvarado and W. Guevara}

Dirección de Astrofísica Comisión Nacional De Investigación y Desarrollo Aeroespacial

Lima, Perú

E-mail: lotiniano@conida.gob.pe

\section{for The LAGO Colllaboration}

lagoproject.org/collab.html

The space weather program of the LAGO project aims to study the galactic cosmic ray modulation estimated from the measurement of secondary cosmic ray flux in the network of detectors of the project. The experiment consists of several water Cherenkov tanks located at different altitudes, ranging from sea level (Lima) up to 5000 meters above sea level (Chacaltaya), and latitudes, which span most of Latin America from $18^{\circ} 59^{\prime} N$ (Sierra Negra) to $41^{\circ} 09^{\prime} S$ (Bariloche). The operation of a detector in the Antarctica is also being considered.

In order to monitor the flow of secondary cosmic rays, a dedicated web monitor has been developed. The monitor automatically calibrates the flow of charge deposited in the detectors every hour, correcting for the long term loss of sensitivity as well as for pressure. The cosmic ray flux, measured by the detectors in bands dominated by different types of particles, is shown hourly.

We show details of the automatic semi-analytic calibration method that was developed and the web monitor operation.

35th International Cosmic Ray Conference - ICRC2017

10-20 July, 2017

Bexco, Busan, Korea

* Speaker. 


\section{Introduction}

The LAtinoamerican Giant Observatory (LAGO) [1] collaboration has built an extensive network of Water Cherenkov Detectors (WCD) operated by a non-centralized collaborative network of Universities and Research Institutes in Latin America and Spain. The Space Weather program of LAGO [3] is oriented to study of the variations of the flow of secondary particles at ground level, that reflect the short and long term modulations of galactic cosmic rays, providing information of the near space environment to Earth.

The detectors of the LAGO network are manufactured with commercial cylindrical plastic tanks purchased at each site [5] with different radious $(R)$ and height $(H)$ containing a volume of purified water, on top a large photomultiplier tube collects the Cherenkov radiation produced in the water by secondary cosmic rays traversing the detector. The detector is light isolated with different coatings. In addition each site implements different methods of purifying the water of the detectors depending on the local facilities. All this causes the signal produced in the detectors by secondary cosmic rays to have appreciable variations. The WCD signals are shaped and digitized by a custom made $40 \mathrm{MHz}$ electronic board controlled by a Digilent Nexys2 FPGA [9].

As tool for the LAGO project's Space Weather program a real-time system that provides information from the project WCD is necessary. The difficulty is to develop a calibration process that is fast and adaptable to possible short-term and long-term variations in signal due to detectors differences, electronic drift, loss of transparency of water, loss of gain of photomultipliers, etc.

The current LAGO calibration process locates the secondary maximum of the total charge spectrum generated in the detector by the secondary cosmic rays. In this paper we explain a semianalitic method to estimate the charge spectrum of the vertical muon from the total spectrum, then we use this spectrum to define bands of higher electromagnetic, muonic, or multiparticle content in the spectrum. Finally apply this method to monitor the behavior of one WCD from the LAGO network.

\section{Semianalitical Vertical Muon Equivalent Charge Spectrum Calculation}

The LAGO project has developed CORSIKA based tools to simulate the radiation environment of sites of it's detectors, the simulations of each site are currently being developed[3]. We will take as a case study the Huancayo (HYO) detector [4]. The simulations show that the flow of muons reaching the detector has an energy above $100 \mathrm{MeV}$ (figure 1), so we will assume that muons traverse the volume of the water without interacting and with deprecated deflections.

Using the background simulations we calculate the expected one hour track lenght $\left(T_{L}\right)$ distributions of muon traversing the detector. As expected the peak is reached at the detector height also could be notice that the distribution approaching well to the ideal case of an infinite with $\mathrm{R}>>2 \mathrm{H}$ [7] (Figure 2).

The charge of a WCD is obtained by time integration of the individual pulses measured in the WCD (in ADC units). In order to calibrate the detector we must calculate the expected charge distributions produced in the detector by the VEM. It can be approximated to a gaussian distribution as follow: 


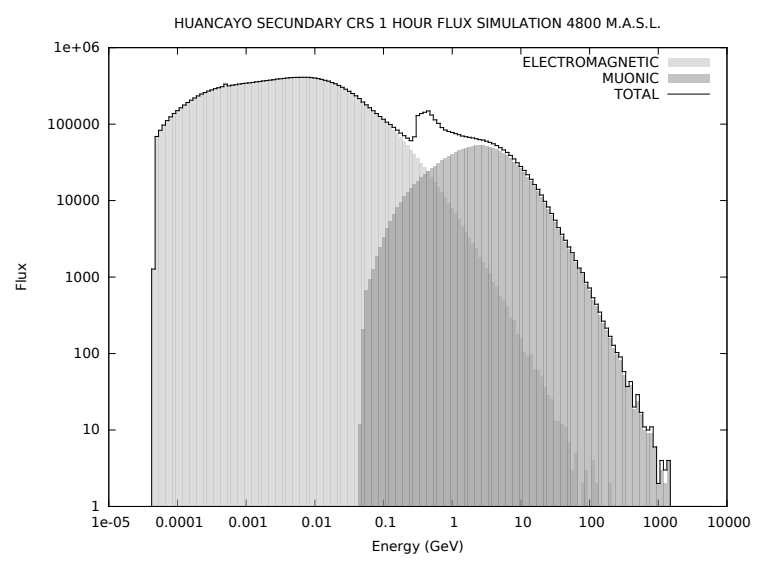

Figure 1: Simulated secondary cosmic ray spectrum in the location of the OMH at 3300 m.a.s.l. We show the main components of the flux (electromagnetic and muon).

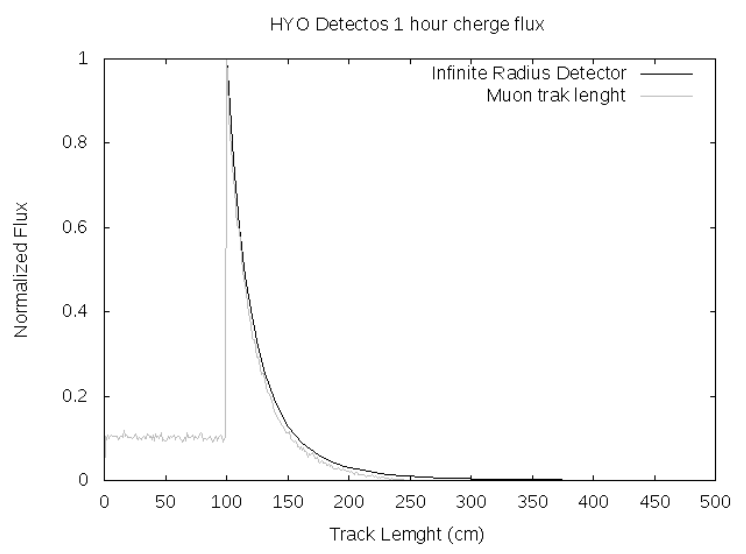

Figure 2: Simulated normalized one hour track length distributions (grey) of muons going through HYO detector with height $(H=90 \mathrm{~cm} R=1,20 \mathrm{~cm})$ and infinite surface model (black)

$$
V^{V E M}(q) \propto e^{-\frac{\left(q-q_{0}\right)^{2}}{2 S_{0}^{2}}}
$$

From [7] the charge distribution is given by:

$$
F(q)=\int_{0}^{\sqrt{H^{2}+4 R^{2}}} V\left(q, T_{L}\right) \frac{d n\left(T_{L}\right)}{d T_{L}} d T_{L}
$$

where $V\left(q, T_{L}\right)$ is the charge distribution for a track lenght, again following [7] we can obtain it from the $V E M$ distribution $V^{V E M}(q)$ :

$$
V\left(q, T_{L}\right)=\frac{H}{T_{L}} V^{V E M}\left(q \frac{H}{T_{L}}\right)
$$

In a previous work [8] we show that using 2.2 and 2.3 we obtain a expression that relates the $V\left(q, T_{V E M}\right)$ and $F(q)$ : 


$$
F(q)+\frac{q}{5} F^{\prime}(q) \propto V^{V E M}(q)
$$

So this allows us to estimate the VEM [6] distribution using the charge distribution measure in the detector. Previous calibration methods estimate the peak in the of $V^{V E M}(q)$ directly from the secondary peak of the charge distribution not taking account the second therm of the left side of equation 2.4 (from now the left side will be call trak correction).

\subsection{The Huancayo Detector}

The muon and electromagnetic components in the charge distribution of Huancayo detector are superimposed (figure 3), we apply the selection described in [10], in order t obtail the muon charge histogram. this selection uses the fact that the muonic signal has higher peaks than the electromagnetic component, compares two consecutive pulses and discard the one that have smaller peak.

In figure 3 we also show the track correction, calculated from the muon selection charge histogram, after a soften the derivative.

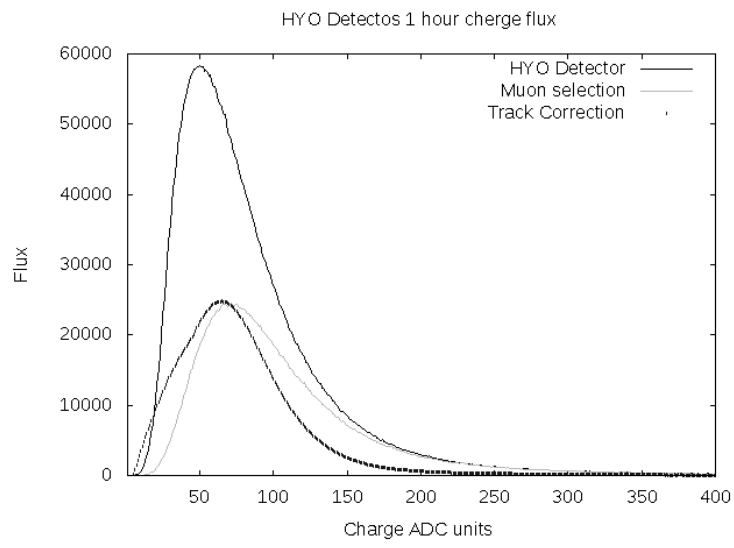

Figure 3: One Hour charge histogram HYO detector (Black), muon selection from first (grey) and muon track correction (dots)

By making a parameter adjustment in equation 10 in a range around the maximum we obtain $q_{0}=65 \mathrm{ADC}, S_{0}=30 \mathrm{ADC}$ for HYO detector. In order to validate the method we will use 2.2 to estimate the total muonic contribution of the 1-hour secondary simulation on HYO. Figure 4 shows the results that are in good agreement charge histogram, particularly it reproduce the muon selection peak and the teh left slope.

\section{The web monitor of the LAGO-project}

Performing a similar process described in the previous section and following [8] we estimate the charge distribution produced by VEMs and use it to define three bands in the total charge histogram (as in [3]) of the Lima, Peru detector figure 5 ). An intermediate band dominated by 


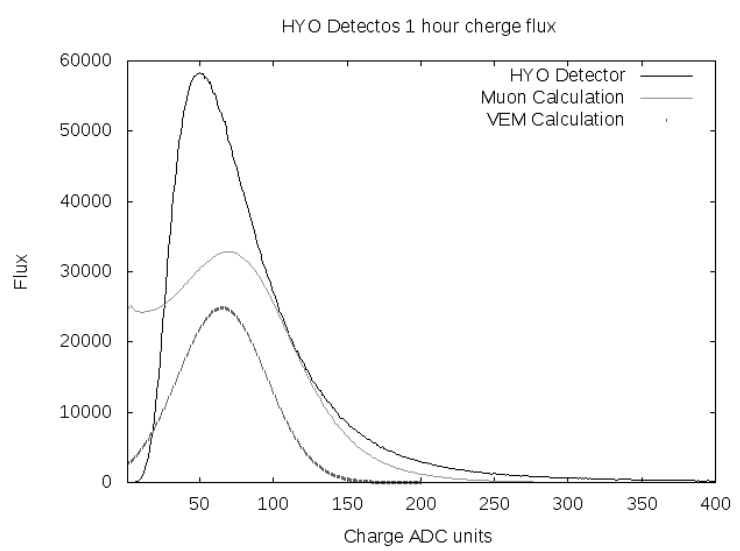

Figure 4: One Hour charge histogram HYO detector (Black), compared to total muon calculation (grey) and VEM contribution calculation(dots)

muons, higher values correspond to simultaneous entrance of multiple particles, while lower values are dominated by electromagnetic compound $\left(\gamma\right.$ and $\left.e^{ \pm}\right)$

In figure 5 we show captures of the web monitor developed at cloud platform, that implement the described analysis on the data of the detector hourly and display it. In figure 6 captures of another available tools via password are show.

\section{Discussion}

The analysis and results of the two detectors in Peru and a third in [8] show that we have a viable method for the rapid calibration of the data of the LAGO network detectors. In addition, the method must be validated, for this more detectors must be analysed and independent measures of VEM of histograms must be obtained.

\section{Acknowledgments}

The autors gratefully acknowledges the financial support from Innovate Perú: Fondo para la Innovación, Ciencia y Tecnología (FINCyT) trough the project PIBA-2-P-020-14. The LAGO Collaboration is very thankful to the Pierre Auger Collaboration for its continuous support.

\section{References}

[1] H. Asorey for the LAGO Collaboration. LAGO: the latin american giant observatory. Nuclear Instruments and Methods in Physics Research Section A: Accelerators, Spectrometers, Detectors and Associated Equipment, 2017.

[2] D. Heck et al. CORSIKA : A Monte Carlo Code to Simulate Extensive Air Showers. FZKA,6019:1-98, 1998.

[3] M.S. Durán, H. Asorey, S. Dasso, L. Nuñez, Y. Peréz, C. Sarmiento for the LAGO Collaboration. The LAGO space weather program: Directional geomagnetic effects, background fluence calculations and 
multi-spectral data analysis, The 34th International Cosmic Ray Conference Vol. PoS (ICRC2015) (p. 142).

[4] Otiniano, L., et al. Development of a high altitude lago site in Peru. The 34th International Cosmic Ray Conference, Vol. PoS (ICRC2015). (p. 688).

[5] I. Sidelnik for the LAGO Collaboration The Sites of the Latin American Giant Observatory, The 34th International Cosmic Ray Conference Vol. PoS (ICRC2015). 2015. p. 665.

[6] J. Ridky, et al. The surface detector of the Pierre Auger Observatory. Nuclear Physics B (Proc. Suppl.), 2007.

[7] Etchegoyen, A., et al. Muon-track studies in a water Cherenkov detector. Nuclear Instruments and Methods in Physics Research Section A: Accelerators, Spectrometers, Detectors and Associated Equipment 2005, vol. 545, no 3, p. 602-612.

[8] L. Otinino, A., et al. VEM Semianalitycal Correction in LAGO water Cherenkov detector. In preparation

[9] Haro, M. Sofo, L. H. Arnaldi, et al. The data acquisition system of the Latin American Giant Observatory (LAGO). Nuclear Instruments and Methods in Physics Research Section A: Accelerators, Spectrometers, Detectors and Associated Equipment 820 (2016): 34-39.

[10] Salazar, H., and L. Villasenor. Separation of cosmic-ray components in a single water Cherenkov detector. Nuclear Instruments and Methods in Physics Research Section A: Accelerators, Spectrometers, Detectors and Associated Equipment 553.1 (2005): 295-298. 


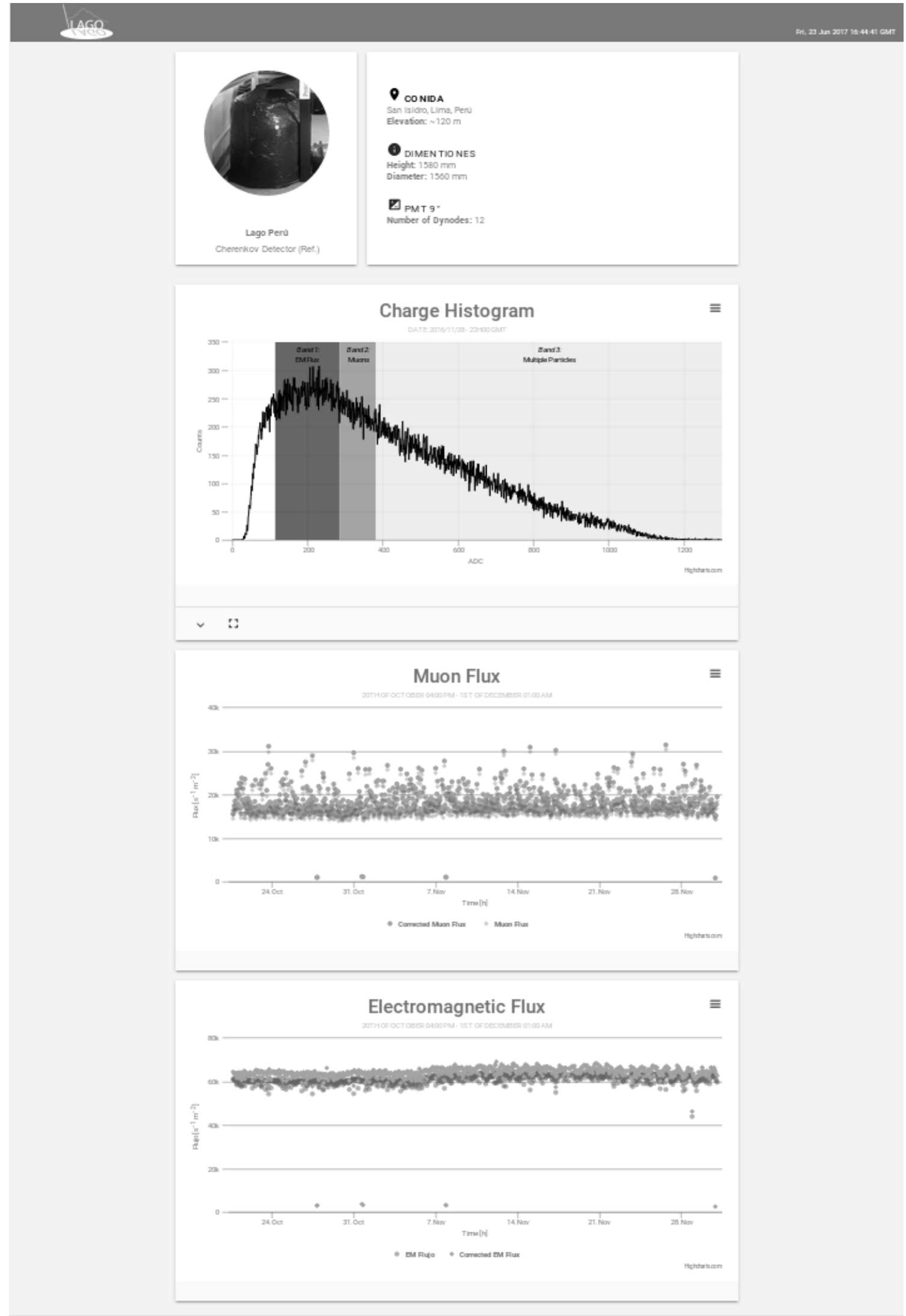

Figure 5: Print screen of the web monitor developed for the detectors in the LAGO project, top-down: detector characteristics, particle flow divided into bands, total flow in the muon band hourly, total flux in the electromagnetic band hourly. 


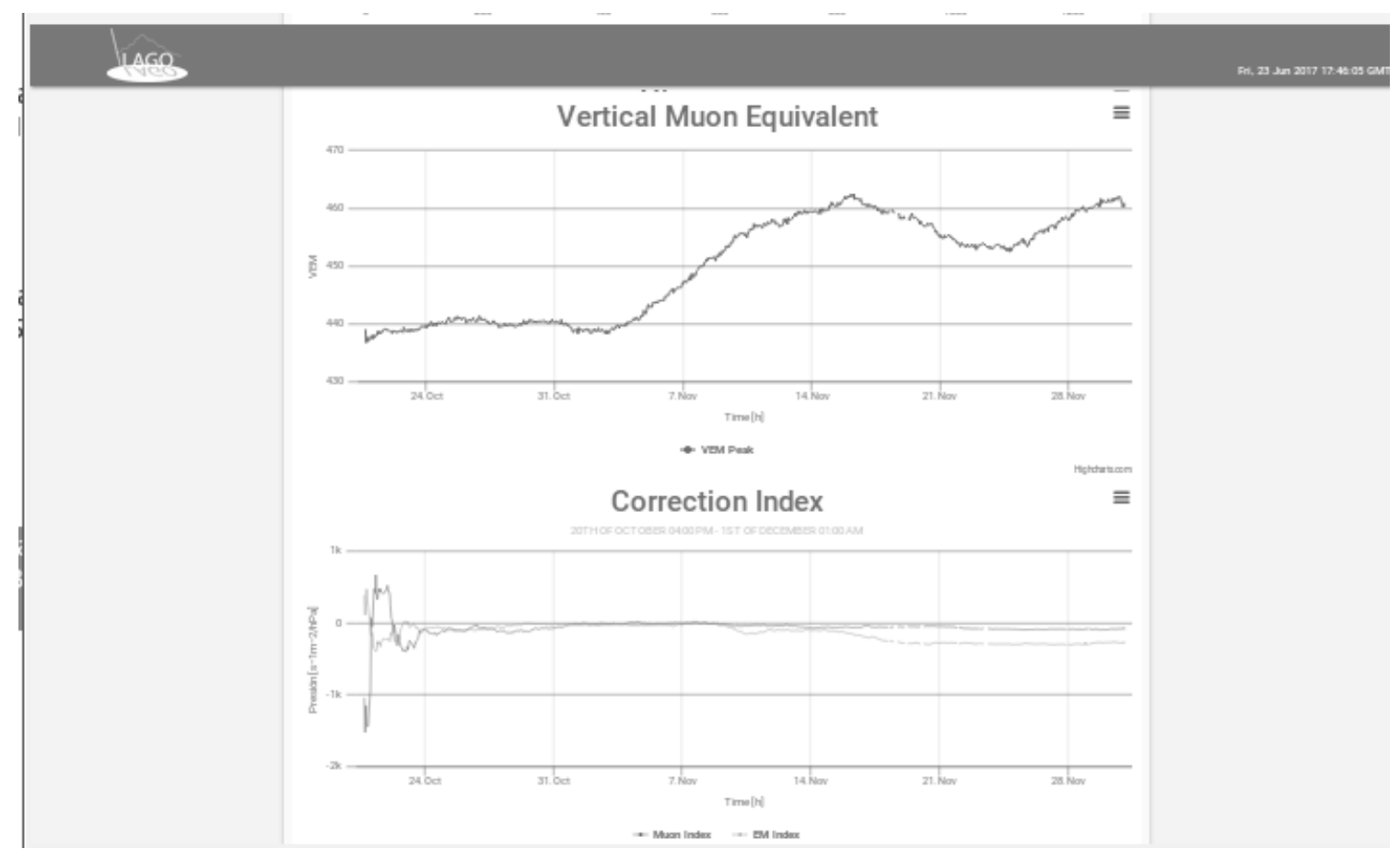

Figure 6: Print screen of the private information of the web monitor, top-down: VEM value hourly, pressure correction index hourly. 